\title{
Antituberculotic Chemotherapy-General and Hepatic Toxicity Revisited
}

\author{
Ali Akbar Sial, Aisha Jabeen, Talha bin Fayyaz, Maria Muneer, Rabia Bushra*, Nusrat Bano, Mirza Tasawer Baig \\ Faculty of pharmacy, Ziauddin University, Karachi, Pakistan.
}

\author{
ARTICLE INFO \\ Article history: \\ Received on: 27/09/2013 \\ Revised on: $23 / 11 / 2013$ \\ Accepted on: 14/12/2013 \\ Available online: $30 / 01 / 2014$ \\ Key words: Antituberculotic \\ Chemotherapy, Hepatic \\ Toxicity, isoniazid, \\ rifampicin and pyrazinamaide
}

\begin{abstract}
Severe or pertinent hepatic toxicity interferes with antituberculotic chemotherapy resulting in dose reductions, treatment delays or cessation of therapy. Hepatic toxicity by antituberculotic agents is due to anaphylactic reactions (acetylaor phenotype polymorphism) and is relative to the cumulative dose intensity. Risk of hepatic toxicity is higher in the elderly and alcoholic patients. Patients with previous hepatic diseases such as hepatitis and comorbidities i.e. HIV infections, malnutrition and renal damages are prone to an added risk of hepatic toxicity. This review consolidates the pattern of hepatic adverse effects associated with each component of the antimyobacterial regimen e.g. isoniazid, rifampicin and pyrazinamaide. Higher propensities of hepatic adverse effects are associated with the first line agents, intensified by the incorporation of second line antibiotics, primarily metabolized in the liver. In conclusion the hepatic biomarkers should be monitored in the patient under a tuberculosis treatment plan as well as purposefully assessed during follow-up visits of the patients.
\end{abstract}

\section{INTRODUCTION}

Tuberculosis (TB) is a global health problem and considered as a second leading cause of death. It is estimated that1.4 million (99,000 among HIV negative people and 430,000 HIV associated) deaths are due to tuberculosis worldwide (Mario et al., 2012).TB is a contagious disease, caused by Mycobacterium tuberculosis (Flynn et al., 1993), which invades the lungs and also infects organs like gastro intestinal tract (GIT), skin, genitourinary tract, and brain lymphatic system (Muñoz et al., 2005). Clinical signs and symptoms of TB include; loss of body mass due to anorexia, fatigue, lethargy, and fever with chills. Patients may also develop purulent cough with chest pain and bloody sputum (hemoptysis) while coughing or breathing (Flynn et al., 1993).

\section{Transmission and Pathogenesis}

The disease is transmitted by sneezing, speaking, singing and pitting mucus discharge (Flynn et al., 1993). The droplets containing tubercle bacilli transmit to other persons by nasal passage (Bavin, 1949). Via inhalation, tubercle bacilli make their passage to pulmonary alveoli and their replication within

\footnotetext{
* Corresponding Author

Email: rabia_pharmacist@hotmail.com
}

macrophages is initiated (American Thoracic Society and CDC, 2000). These replicated mycobacterium are released on destruction of the macrophages (American Thoracic Society and CDC, 2000). In live macrophages, bacilli are transported through blood or lymph to tuberculosis susceptible sites (American Thoracic Society and CDC, 2000). Macrophages are then gathered around the infected cell, forming a structure called granuloma (or fatty metamorphosis) to prevent dissemination of infection (American Thoracic Society and CDC, 2000).

Granuloma consists of central area of serous necrosis with epitheloid macrophages and lymphocytes at the margin (Hunter, 2011).Centre of granuloma undergoes calcification and converting into Ghon's complex cavities are hence form by softening and fragmentation of necrotic mass so as large clumps are coughed out damaging the lungs (Hunter, 2011).

\section{How Tuberculosis Influences the Quality of life (QOI)}

Quality of life is a person's perception of his/her wellbeing. According to a study conducted in 2008, TB mainly affects physical and psychological health of individuals, creating socioeconomic burdens and stress. Women have better QOL in physical and environmental domain whereas men have better economic and social health as compared to women. 
Patient's sense of security; safety, domestic environment, transport and financial security are also adversely affected due to this disease (Dhuria et al., 2008).

\section{First line Chemotherapy of Tuberculosis}

The first line agents for active tuberculotic infections are isoniazid (INH), rifampin (RMF), Ethambutol and pyrazinamide (PZA), with a broad spectrum of adverse effects (American thoracic society \& CDC, 2003, Combs et al., 1990, Long and Ellis, 2007). Isoniazid (INH) is the mainstay in TB treatment since 1952. The structure comprises of hydrazine group and a pyridine ring (Jindani et al., 2004, Kass and shandera, 2010). It is a pro-drug, activated by tuberculosis catalase-peroxidase enzyme (Kat G) and results in production of per oxy nitrate and hydrogen peroxide (both are oxygen free radical). These radicals inhibit the mycolic acid, responsible for DNA damage and finally the death of the bacillus (Kass and Shandera, 2010, Flynn et al., 1993).

\section{Isoniazid}

INH causes severe hepatic toxicity as it is metabolized in the liver by $\mathrm{N}$-acetyl transfers by acetylation, produces isonicotinic acid and acetyl INH. It is mainly (70-90\%) excreted by kidney mostly in feces (Singh et al., 2012). In the first phase, it is metabolized by $\mathrm{N}$ - acetyl tranferase to acetyl isoniazid which is then hydrolysed to acetylhydrazine (Niemi et al., 2003). A small portion of drug is directly hydrolyzed into isonicotinic acid and hydrazine (Baghaei et al., 2010). Drugs at a dose of 10-300 $\mathrm{mg} / \mathrm{kg} /$ day are used for prophylaxis of TB, such doses could rarely cause adverse effects in individuals having regular kidney and liver functions. Few uncommon adverse effects include; nausea, vomiting, and epigastric pain (initiated at chemoprophylaxis). However, symptoms can be overcome by metoclopramide, ranitidine or omeprazole after the first meal (Tai et al., 2008). High levels of hepatic enzymes are usually assessed, which may be transitory and asymptomatic. Alanine aminotransferase ALT (glutamic -pyruvic transaminase) increases three folds in comparison to normal serum level and it particularly caused liver damage (American thoracic society \& CDC, 2003, Jindani et al., 2004, Moulding et al., 1989). Arthralgia behavioral changes like headache, euphoria, insomnia, agitation, and anxiety are the other symptoms, may overcome on discontinuation of therapy (Kass and Shandera, 2010, Baghaei et al., 2010).Major side effects of Isoniazid are psychosis, seizures, convulsions, coma and mental disturbances which are not common and difficult to assess. Differential diagnosis of symptoms also indicates meningitis and hepatic encephalopathy. In addition, suicidal attempts also reported in patients using INH (Campos-Franco et al., 2004). Peripheral neuropathy occurs in about $20 \%$ of patients treated at dose more than $30 \mathrm{mg} /$ day. Risk of polyneuritis increases in individuals having diabetes mellitus, alcoholism, advanced age nutritional deficiency, kidney failure, pregnancy and breast feeding (Combs et al., 1990, Kass and Shandera, 2010, Forget and Menzies, 2006). Development of hepatitis has been dependent on the age of patients, and it is considerably rare in patients that are under 20years but increases up to $2 \%$ in 50-64 years old patients (Campos-Franco et al., 2004). Isoniazid has better absorption in acidic medium. Carbohydrates are responsible to decrease the absorption of drug up to $57 \%$ and $30 \%$ of plasma concentration. INH is a potent inhibitor of monoamine oxidase enzyme, should not be taken with glucose or lactose fluids and foods rich in histamine and tyramine like cheese, fish, alcohol and red wine. Otherwise, interaction may produce symptoms like flushing of face, erythema, headache, palpitation and pruritus. INH increases the plasma concentration of certain drug like phenytoin, carbamazepine (Zhang and Yew, 2009). Absorption also decreases by those drugs that increasing gastric $\mathrm{pH}$. It is recommended that antacids containing ranitidine and aluminum hydroxide should be taken after one hour of INH administration.

\section{Rifampin}

Rifampin is used for the treatment of tuberculosis since 1966. It possesses bactericidal action and kills metabolically active bacilli as well as stationary bacilli (having slow metabolism) (Singh et al., 2012). It inhibits RNA synthesis of bacteria, by strong binding to $\beta$ subunit of bacterial "DNA- dependent RNA polymerase". This $\beta$ subunit of the polymerase enzyme is necessary for the initiation and growth of RNA chain (Kass and Shandera, 2010, Yew, 2002).It is well absorbed after oral administration. It is mainly excreted by liver ( $85 \%$ ) into bile while, $6-15 \%$ is excreted unchanged and reabsorbed by intestine (Zhang, 2005). It has ability to cross blood brain barrier, and about $10-20 \%$ of serum concentration reaches to CNS. However, concentrations may increasing during meningitis (Combs et al., 1990).Minor adverse reactions include; nausea, anorexia, fatigue, dyspnea, dizziness, ataxia, abdominal pain, and change of urine's color from yellow to orange. The risk of hepititis is augmented in patients, with compromised hepatic status, treated with INH. It is a potent inducer of CYP450 system, results in increased metabolism of all those drugs metabolized by the same enzyme system (Kalars et al., 1992, Combalbert et al., 1989). Increased production of bilirubin (5\% of patients), and development of cholestatic hepatitis $(2.7 \%$ of patients) have been reported (Baciewicz et al., 2008, Zhang and Yew, 2009).Immunological reactions are rare but severe in less than $0.1 \%$ of patient. Other reactions include; thrombocytopenia, leukopenia, eosinophilia, agranulocytosis, vasculitis, hemolytic anemia, acute interstitial nephritis and septic shock (Combs et al., 1990, Kass and Shandera, 2010).In presence of food plasma concentrations are reduced up to $26 \%$ as well as delaying of the time $(15-36 \%)$ to reach the maximum concentration (World Health Organization, 2010). It is a potent inducer of the cyp450 system and its sub families like cyp3A and cyp2C, resulting in increased the metabolism of many drugs (Slayden and Barry, 2000, Handbook of antitubercolusis agents, 2008).

\section{Pyrazinamide (PZA)}

PZA (pyrozoic acid amide) is a nicotinic acid derivative and structurally similar to INH. It has been used for the treatment of tuberculosis since 1936. It possesses potent bactericidal and 
sterilizing effect and shortens the duration of treatment (Combs et al., 1990, Rakotoson et al., 2009).It accumulates to decrease intracellular $\mathrm{pH}$, resulting in deactivation of an enzyme fatty acid synthatase 1. This enzyme is involved in fatty acid synthesis, leading impaired biosynthesis of mycolic acid (Handbook of antitubercolusis agents, 2008).

It is mainly metabolized by the liver and $70 \%$ drug is excreted in urine through glomerular filtration. The half-life is 910 hours, but dose adjustment is required in renal compromised patients, otherwise the half- life may be raised up to 26 hours (Combs et al., 1990, Kass and Shandera, 2010).Minor side effects include vomiting, nausea and anorexia (American Thoracic Society \& CDC, 2003, Kass and Shandera, 2010).If patients are non-gouty then hyper-uricemia leads towards more arthralgia. Dermatitis, exanthema subitum and pruritus are also commonly effects of drugs (American Thoracic Society \& CDC, 2003, Tai et al., 2008). Therapy should be discontinued if severe pruritus and severe exanthema occur (Combs et al., 1990). It possesses prominent hepatotoxic effect so dose should be adjusted according to the weight of the patient. The risk of liver damage decreases, if administered in a dose of $35 \mathrm{mg} / \mathrm{kg} /$ day. According to new guideline (American Thoracic Society \& CDC, 2003, Long and Ellis, 2007) the recommended dose for a patient having more than $50 \mathrm{~kg}$ weight is $1600 \mathrm{mg}$, such dose is associated with reduced hepatic adverse effects. According to WHO PZA is safe in pregnancy, breast feeding (American Thoracic Society \& CDC, 2003, Brunton et al., 2006) and considered as category C drug (Kass and Shandera, 2010, Yew, 2002). Hepatotoxic effect is more evident in those individual having liver diseases (Combs et al., 1990, Tai et al., 2008). Metabolites of drug are eliminated by kidney, dose adjustment is critical in renal compromised patients. It is recommended that if creatinine clearance is lower than $10 \mathrm{ml} / \mathrm{min}$ then dose should not be reduced to half. Food has no impact on absorption (World Health Organization, 2010). Some drugs potentiate the toxic effect of pyrazinamide- like ethionamide, rifampin, isoniazid and propencid (Combs et al., 1990, Yew, 2002, American Academy of Pediatrics Committee on Drugs, 2001).

\section{Ethambutol}

Ethambutol has been in therapeutic use against TB since 1966. The mechanism of action for bacteriostatic effect is still not fully defined. Arabinogalactan is main component of mycobacterial cell wall and Ethambutol interferes with the biosynthesis of arabinogalactan. It prevents arabinoglycons polymerization by inhibition of enzyme arabinosyltranferase that encoded EmbB gene. If mutation occur in EmbB gene then resistance would developed against ethambutol (Combs et al., 1990, Rakotoson et al., 2009).It is absorbed up to $75-80 \%$ after oral administration and serum peak plasma concentration is achieved within 2-4 hours. The half- life of drug is 3-4 hours, extended in renal compromised patients (up to 10 hours). It is metabolized by liver and $50-80 \%$ drug is excreted in urine and $20 \%$ in feces (American Thoracic Society \& CDC, 2003, Brunton et al., 2006, Yew, 2002).It does not cross the meninges but in meningitis the cerebral spinal fluid (CSF) level of drug reaches up to $10-50 \%$ of the drug plasma level (American Thoracic Society \& CDC, 2003, Brunton et al., 2006, World Health Organization, 2010).Generally drug is well tolerated and adverse effects are dose related and more common at doses of $15 \mathrm{mg} / \mathrm{kg}$. Retrobulbar neuritis usually occurs, but it is reversible, dose and time dependent. If patients received $35 \mathrm{mg} / \mathrm{kg} / \mathrm{day}$, for more than two months, it results in development of retrobulbar neuritis (15-18\% of patients). In young children ethambutol should be avoided because of red and green color blindness (Combs et al., 1990, Brunton et al., 2006, Yew, 2002). Peripheral neuritis occurs rarely and symptoms can be improved by administration of pyridoxine (American Thoracic Society \& CDC, 2003, Brunton et al., 2006, Yew, 2002). Additional effects are abdominal pain, nausea, vomiting and hepatotoxicity. Hematological symptoms include; thrombocytopenia, eosinophilia and neutropenia while neurological symptoms like headache mental confusion and dizziness are also found. Hypersensitivity like fever, arthralgia and skin rash may also develop. It may decreases excretion of uric acid from kidney which leads toward hyperurecemic gouty arthritis (American Thoracic Society \& CDC, 2003, Brunton et al., 2006, Yew, 2002). According to WHO recommendations, it is safe in pregnancy, infant lactation, and considered as category B drug (World Health Organization, 2010). It does not produce any toxicity in liver failure patient and so no need to adjust the dose of drug (American Thoracic Society \& CDC, 2003, Combs et al., 1990). However, dose should be adjusted in patients of kidney impairment. Dose should be reduced to $15-20 \mathrm{mg} / \mathrm{kg}$ thrice a week if creatinine clearance is lower than $30 \mathrm{ml} / \mathrm{min}$ and if Creatinine clearance is measured within $30-50 \mathrm{ml} / \mathrm{min}$, dosing interval should be extended up to 36 hours (Combs et al., 1990, Jindani et al., 2004, Kass and Shandera, 2010, Tai et al., 2008).

\section{Second \&Third Line Drugs}

These are used in case of XDR-TUBERCULOSIS or MDR-TUBERCULOSIS.

Six classes are included.

1. Aminoglycosides(streptomycin,amikacin,kanamycin)

2. Polypeptides (capreomyccin, viomycin,enviomycin)

3. Flouroquinolones(ciprofloxacin,ofloxacin,levofloxacin,m oxifloxacin,gatifloxacin,)

4. Thioamides (ethionamide, prothionamide,Cycloserine)

5. Terizidone

6. Para aminosalicylic acid

7. Oxazolidones and its analogues (Linzolide, eprezolide) (Pandit et al., 2012)

\section{Ethionamide (EthA)}

Ethionamaide is always used with other anti-tuberculosis drugs for MDR-TUBERCULOSIS when first line drugs are contraindicated. It is also effective in lepromatous leprosy. It can cause temporary asymptomatic increase in ALT, AST and acute 
liver injury in $5 \%$ of patients is also reported (Tarantino et al., 2009).It is a pro-drug, similar to INH, metabolized by mycobacterial enzyme EthA for activation. Upon activation, it inhibits the formation of mycobacterial cell wall by inhibiting mycobacterial fatty acid synthesis, mediated by enoyl ACP reductase (Tarantino et al., 2009). It inhibits the growth of both extracellular and intracellular bacilli (Arbex et al., 2010). Dose of ethionamide is $250 \mathrm{mg}$ twice daily, could be enhanced to $15-20$ $\mathrm{mg} / \mathrm{kg} / \mathrm{day}$, with the maximum dose up to $1 \mathrm{~g} /$ day (Arbex et al., 2010).Adverse effects are gastrointestinal (anorexia, nausea, stomatitis, diarrhea), CNS (depression, drowsiness, fatigue, hallucinations, confusion), endrocrinological disturbances (gynecomastia,hypothyiodism), cardiovascular and skin conditions(decrease platelets count, pupura), swelling in feet and weight gain (Arbex et al., 2010) .Ethionamide can cause selflimited and asymptomatic increase in serum transaminases. It also causes fatal acute liver injury. It possess similar onset of action and clinical manifestations as that of INH. Hypersensitivity reactions are rare (Tarantino et al., 2009). Metabolism takes place in liver for activation of drug and result in toxic and immunologically active intermediate and hepatitis. Transaminase elevation is observed in treatment induced hepatitis 5 times higher than normal (Tarantino et al., 2009). Strict monitoring is advised in patients underlying liver diseases (Arbex et al., 2010).

\section{Paraamino Salicylic Acid (PAS)/D5 Amino Salicylic Acid}

For more than a decade, PAS was thought to be first line drug, used in combination with INH and streptomycin.PAS inhibits the growth of extracellular bacilli. Liver and kidney damaged are associated with oral or parenteral administration of drug for prolonged period of time (Bavin, 1949). The proposed mechanism of drug is interruption of bacterial tetrahydrofolate formation and inhibition of iron uptake, folate and iron are essential components for bacterial growth and life (Arbex et al., 2010). It causes immune induced hepatitis which generally initiated in first 3 weeks of therapy. Clinical symptoms include rashes, fever, conjunctivitis; hepatomegaly, leukocytosis, lymphadenopathy and eosinophilia.5 to $10 \%$ of patients come across hypersensitivity reactions which usually occurred in first 5 weeks of the therapy. PAS causes cholistatic hepatitis, elevation in transaminases, cirrhosis, liver necrosis and liver failure.

\section{Aminoglycoside}

Streptomycin is a natural derivative; kanamycin is synthetic while amikacin is semi-synthetic aminoglycosides (Arbex et al., 2010). These agents are protein synthesis inhibitors, acting through binding to 30s subunit of mycobacterial ribosome in irreversible fashion (Arbex et al., 2010). Resistance develops as a result of mutation in "rrs" "nad" and "rpsl" genes, encoded for S12 ribosomal RNA and S16 ribosomal protein (Arbex et al., 2010). Oral absorption of aminoglycosides is poor so generally administered by intramuscular injection, and plasma peak concentrations are achieved within 30 to 90 minutes. These drugs possess low plasma protein binding and are almost excreted by kidney through glomerular filtration (Arbex et al., 2010). Ototoxiciy is reported due to vestibular and cochlear damage. Vestibular damage associated with streptomycin is more common than amikacin. Nephrotoxicity (reduced urination, proteins in urine, and reduced creatinin clearance)is highly reported with amikacin as compared to streptomycin (Arbex et al., 2010). Aminoglycosides are contraindicated in pregnancy and breast feeding mothers since it disturbs the intestinal flora of neonates. Amphoteracin B, vancomycin, loop diuretics (furosemide and ethacrinic acid) potentiate the nephrotoxicity of aminoglycosides (Arbex et al., 2010).

\section{Fluoroquinolones}

These include oflaxacin, levofloxacin, ciprofloxacin and others. These agents have been used as second line drugs for the treatment of multi drug resistance tuberculosis while moxifloxacin for extensive drug resistant tuberculosis (Yew and Leung, 2006). These agents penetrate inside intracellular mycobacteria and macrophages. DNA gyrase or topoisomerase II of Mycobacterium tuberculosis maintains DNA topology and essential for its life. These drugs inhibit such enzymes leading to cell death due to uncontrolled mRNA synthesis and chromosomal degradation (Arbex et al., 2010).

Fluroquinolones are actively absorbed after oral administration. Peak serum concentration is achieved within 3 hours. Levofloxacin is converted to d-ofloxacin in liver. Fluroquinolones are excreted mainly by kidney through tubular secretion or glomerular filtration (Arbex et al., 2010). Adverse effects include gastrointestinal complains, neuro-toxicities, cardio vascular problems, musculo-skeletol disorders, and endocrinological irregularities (Arbex et al., 2010).

\section{CONCLUSIONS}

Substantial risk of hepatic toxicity is associated with the first line agents employed for the treatment plan of patients with active disease. Hepatic toxicity is correlated with the elevation of serum transaminases, which should be objectively assessed during the course of treatment. The abnormal rise in enzyme levels (3-5 $x$ of normal values) may require cessation of therapy even in the absence of symptoms. The hepatic status should also be assessed in the patients during follow-ups.

\section{REFERENCES}

American Thoracic Society and CDC. Diagnostic standards and classification of tuberculosis in adults and children. Am J RespirCrit Care Med,2000, 161 (4), 1376-1395.

American Thoracic Society/Center for Disease Control Prevention / Infectious Disease Society of America. Treatment of Tuberculosis. Am J RespirCrit Care Med ,2003,167:603-662.

American Academy of Pediatrics Committee on Drugs. Transfer of drugs and other chemicals into human milk. Pediatrics, 2001,108 (3),776-89.

Arbex MA, Varella MCL, Siqueira HR, Mello FAF. Antituberculosis drugs: Drug interactions, adverse effects,and use in special situations,. J Bras Pneumol, 2010, 36(5),641-656. 
Baciewicz AM, Chrisman CR, Finch CK, Self TH. Update on rifampin and rifambutin drug interaction. AM J Med Sci, 2008, 335(2), 126-36.

Baghaei P, Tabarsi P, Chitsaz E, Saleh M, Marjani M, Shemirani S. Incidence, clinical and epidemiological risk factors and outcome of drug-induced hepatitis due to antituberculous agents in new tuberculosis cases. Am J Ther,2010,17(1),17-22.

Bavin EM. Some Aspects of the Pharmacology Of Para-Aminosalicylic Acid. J Pharm Pharmacol, 1949,1(1),790-810.

Brunton LL, Lazo JS, Parker KL. Goodman\& Gilman - As Bases Pharmacologicas da Terapeutica. Rio de Janeiro: McGraw-Hill; 2006, 1083-1101.

Campos-Franco J, Gonzalez-Quintela A, Alende-Sixto MR. Isoniazid-induced hyperacute liver failure in a young patient receiving carbamazipine. Eur J Intern Med.2004,15(6),396-7.

Combalbert J, Fabae I, Fabne G. Purification and identification of the rifampicin-inducible human liver cytochrome P-450 (cyclosporine A oxidase) as a product of P-450 III A gene subfamily. Drug Metab.Dispos, 1989,17, 197-207.

Combs DL, O' RJ, Geiter LJ. USPHS Tuberculosis Short Course Chemotherapy trial 21;effectiveners , toxicity and acceptability ;the report of final results. Ann Inter med, 1990, 112,397-406

Dhuria M, Sharma N, Ingle GK. Impact of Tuberculosis on the Quality of Life. Indian J Community Med, 2008,33(1), 58-59.

Forget EJ, Menzies D. Adverse reactions to first line antituberculosis drugs. Expert Opin Drug Saf, 2006, 5(2),231-49.

Flynn JL, Chan J, Triebold KJ, Dalton DK, Stewart TA, Bloom BR. An essential role for interferon gamma in resistance to mycobacterium tuberculosis infection. NJEM, 1993, 178(6):2249-2254.

Handbook of antitubercolusis agents. Tuberculosis, 2008, $88(2), 102(5)$

Hunter RL. Pathology of post primary tuberculosis of the lung: an illustrated critical review. Tuberculosis, 2011,91(6), 497-509.

Jindani A, Nunn Aj, Enarson DA .Two 8- month regimens of chemotherapy for treatment of newly diagnosed pulmonary tuberculosis international multicenter randomized trial. Lancet, 2004, 364, 1244-1251.

Kalars JG, Schmiedlin-Ren P,Schuetz JD, Fang C, Warkinz PB. Identification of rifampin-inducible P-450 III A4(CYP 3A4)in human small bowel enterocytes. J.Clin. Invest,1992, 90, 1871-8.

Kass JS, Shandera WX. Nervous system effects of antituberculosis therapy. CNS drugs, 2010, 24(8), 655-667.

Long R \& Ellis E. Introducing the sixth edition of the Canadian Tuberculosis Standards. Canadian Respiratory Journal: Journal of the Canadian Thoracic Society, 2007, 14(6), 327.

Mario R, Marais B, Floyd K, Lönnroth K, Getahun H, Giovanni B. Migliori, Anthony D. Harries. Scaling up interventions to achieve global tuberculosis control: progress and new developments. The Lancet, 2012,1902-1913.

Moulding TS, Redeker AG, Kanel GC.Twenty isoniazid associated deaths in one state.Am Rev Respir Dis,1989,140 (3),700-5.

Muñoz P, Rodríguez C \& Bouza E. Mycobacterium tuberculosis infection in recipients of solid organ transplants. Clinical infectious diseases, 2005,40 (4), 581-587.
Niemi, Mikko, Janne T. Backman, and Pertti J. Neuvonen. Effects of trimethoprim and rifampin on the pharmacokinetics of the cytochrome P450 2C8 substrate rosiglitazone \& ast. Clinical Pharmacology \& Therapeutics, 2004; 76 (3): 239-249.

Pandit N, Singla RK, \&Shrivastava B. Current updates on Oxazolidinone and its Significance. International Journal of Medicinal Chemistry, 2012.

Rakotoson JL, Randriamanana D, Rakotomizoa JR, Andrianasolo R, Andrianarisoa AC. Severe systemic lupus erythematosus induced by isoniazid. Rev Pneumol Clin,2009,65(6),361-4.

Singh S, Kumar P, Sharma S, MumbowaF, Martin A, and Durier N.Rapid Identification and Drug Susceptibility Testing of Mycobacterium tuberculosis: Standard Operating Procedure for NonCommercial Assays: Part 1: Microscopic Observation Drug Susceptibility Assay v2.4.12J Lab Physicians. 2012, 4(2),101-111.

Singh S, Kumar P, Sharma S, Mumbowa F, Martin Aand Durier N. Rapid Identification and Drug Susceptibility Testing of Mycobacterium tuberculosis: Standard Operating Procedure for Non-Commercial Assays. Part 3: Colorimetric Redox Indicator Assay v1.3.12Lab Physicians. 2012,4 (2), 120-126.

Singh S, Kumar P, Sharma S, Mumbowa F, Martin A, and Durier N. Rapid Identification and Drug Susceptibility Testing of Mycobacterium tuberculosis:Standard Operating Procedure for NonCommercial Assays: Part 2: Nitrate Reductase Assay v1.3.12 J Lab Physicians. 2012, 4(2), 112-119.

SlaydenRA,Barry CE .The genetics and biochemistry of isoniazid resistance in mycobacterium tuberculosis.Microbes Infect, 2000, 2(6),659-69.

Tai WP,YueH,HuPJ.Coma caused by isoniazid poisoning in a patient treated with pyridoxine and hemodialysis.Adv Ther, 2008, 25(10), 1085-1088.

Tarantino G, Di Minno MND, Capone D. Drug-induced liver injury: is it somehow foreseeable?. World journal of gastroenterology, 2009,15(23), 2817.

World Health Organization.The Treatment of Tuberculosis Guidelines. World Health Organization; Geneva, 2010.

Yew WW. Clinically significant interaction with drugs used in the treatment of tuberculosis. Drug saf,2002,25(2),111-33.

Yew WW, Leung CC. Antituberculosis drugs and hepatotoxicity. Respirology, 2006,11, 699-707.

Zhang Y, Yew WW. Mechanisms of drug resistance in Mycobacterium tuberculosis. Int J Tuberc Lung Dis ,2009,13(11) ,1320-30.

Zhang Y. The magic bullets and tuberculosis drug targets. Annu Rev Pharmacol Toxicol, 2005,45,529-64.

\section{How to cite this article:}

Ali Akbar Sial, Aisha Jabeen, Talha bin Fayyaz, Maria Muneer, Rabia Bushra, Nusrat Bano, Mirza Tasawer Baig. Antituberculotic Chemotherapy-General and Hepatic Toxicity Revisited. J App Pharm Sci, 2014; 4 (01): 148-152. 